\title{
HIV-1 outbreak among injecting drug users in Greece, 2011: a preliminary report
}

D Paraskevis, G Nikolopoulos², C Tsiara², D Paraskeva², A Antoniadou³, M Lazanas4, P Gargalianos ${ }^{5}$, M Psychogiou $^{1,6}$,

M Malliori7, J Kremastinou ${ }^{2}$, A Hatzakis (ahatzak@med.uoa.gr) ${ }^{1}$

1. National Retrovirus Reference Center, Department of Hygiene, Epidemiology and Medical Statistics, Medical School, University of Athens, Athens, Greece

2. Hellenic Center for Diseases Control and Prevention, Athens, Greece

3. 4th Department of Internal Medicine, Athens University Medical School, Attikon Hospital, Athens, Greece

4. 3rd Department of Internal Medicine, Hellenic Red Cross Hospital, Athens, Greece

5. 1st Department of Internal Medicine, Athens General Hospital 'G. Gennimatas', Athens, Greece

6. 1st Department of Medicine, Athens University Medical School, Laiko Hospital, Athens, Greece

7. Organization Against Drugs (OKANA), Athens, Greece

Citation style for this article:

Paraskevis D, Nikolopoulos G, Tsiara C, Paraskeva D, Antoniadou A, Lazanas M, Gargalianos P, Psychogiou M, Malliori M, Kremastinou J, Hatzakis A. HIV-1 outbreak among injecting drug users in Greece, 2011: a preliminary report.

Euro Surveill. 2011;16(36):pii=19962. Available online: http://www.eurosurveillance.org/ViewArticle.aspx?Articleld=19962

Article published on 8 September 2011

A significant increase (more than 10 -fold) in the number of newly diagnosed HIV-1 infections among injecting drug users (IDUs) was observed in Greece during the first seven months of 2011. Molecular epidemiology results revealed that a large proportion ( $96 \%$ ) of HIV-1 sequences from IDUs sampled in 2011 fall within phylogenetic clusters suggesting high levels of transmission networking. Cases originated from diverse places outside Greece supporting the potential role of immigrant IDUs in the initiation of this outbreak.

During the first months of 2011, an unprecedented upward shift in the number of newly diagnosed cases of human immunodeficiency virus type 1 (HIV-1) infection among injecting drug users (IDUs) in Greece was noticed. In order to verify the epicentre of the outbreak and to identify unusual patterns of viral transmission, enhanced surveillance and a molecular epidemiology study among IDUs were conducted. This is a brief overview of surveillance data up to 31 July 2011 and of the preliminary results of the molecular epidemiology analysis.

\section{Epidemiological situation in}

\section{Greece between 2000 and 2010}

From 2000 to 2010, between 397 and 653 cases of HIV-1 infection were notified annually in Greece, with the majority of cases in men who have sex with men (MSM) (Figure 1) [1].

The newly reported cases among IDUs ranged from nine to 19 per year during 2000-2010 [2], which corresponded to approximately $1.5-4.5 \%$ of the total HIV-1 infections reported on an annual basis. A distinctive characteristic of HIV-1 transmission in Greece, compared with other southern European countries, was the unusually low number of HIV-1 infections among IDUs [2]. The low level of HIV-1 transmission in IDUs in
Greece was indeed unexpected given the documented sharing of needles and syringes, and the substantial prevalence of IDU-related hepatitis C infection [1].

\section{Epidemiological situation in Greece during the first seven months of 2011}

From 1 January until 31 July 2011, 555 new cases of HIV-1 infection were notified to the Hellenic Center for Diseases Control and Prevention, in the context of the mandatory HIV-1 / acquired immune deficiency syndrome (AIDS) reporting system (Figure 1).

As in the previous years, most of the cases identified this year were men $(n=481 ; 86.7 \%)$ and homosexual contact was the predominant mode of HIV-1 transmission $(n=174)$. Based on the total number of newly

\section{FIGURE 1}

Newly diagnosed cases of HIV-1 infection reported in Greece, 1 January 2000 - 31 July 2011

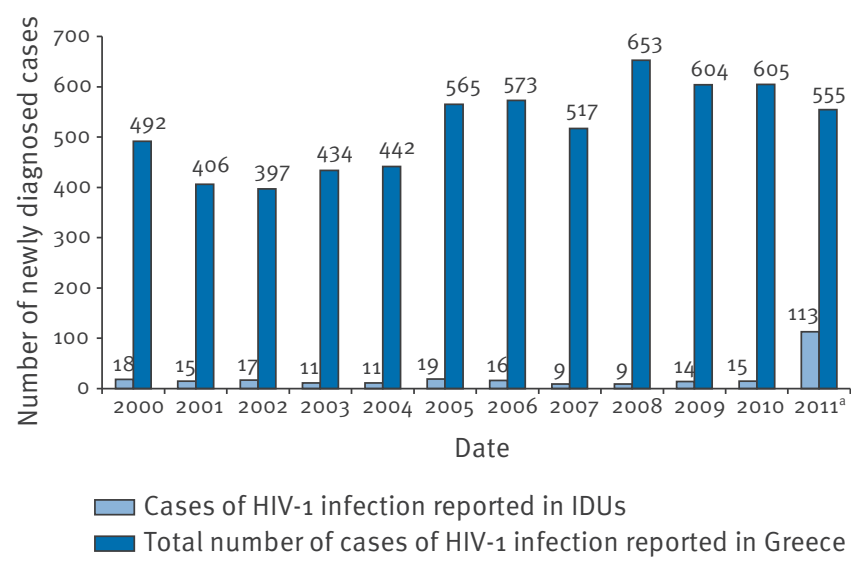

IDUs: injecting drug users.

a 1 January - 31 July 2011. 
HIV-1 diagnosed cases reported until the end of July, we anticipate an approximate increase of $55 \%-60 \%$ in the total annual number of reported cases by the end of 2011.

\section{Transmission among IDUs}

Of the total number of cases, 113 were registered among IDUs (20.4\%), which is the largest number ever reported in this group from the beginning of the epidemic in Greece. This figure represents a more than 10 -fold increase in the number of newly diagnosed cases of HIV-1 infection in IDUs. Among them, $87 \%$ were men, $74 \%$ were aged between 25 and 40 -years old and among the cases for whom the place of residence was known, $76 \%$ came from the Athens metropolitan area. Sixty-seven of the IDU cases were Greek citizens, 18 were foreigners and for 28 the nationality could not be identified. The analysis of preliminary data suggests that IDUs accounted for $50 \%-55 \%$ of the increase in the total number of HIV-1 infection cases reported during 2011.

\section{Molecular epidemiology analysis in IDUs}

To identify the origin and patterns of HIV-1 spread among IDUs, phylogenetic analyses were performed on HIV-1 sequences sampled from newly identified IDUs $(n=34)$ collected from the beginning of 2010 until the end of May 2011. Specifically, 11 plasma specimens collected in 2010 and 23 plasma specimens collected in 2011, submitted for routine HIV RNA and drug resistance testing were analysed. HIV-1 protease (PR) and partial reverse transcriptase $(R T)$ sequences were generated using the HIV-1 TRUGENE Genotyping kit (Bayer, HealthCare) and ViroSeq HIV-1 Genotyping system (Celera Diagnostics). HIV-1 subtypes were determined manually by phylogenetic analysis including a set of reference sequences (http://www.HIV.lanl.gov) and also by using the COMETHIV-1/2 subtyping tool (v. 0.2) (http://comet.retrovirology.lu/). Phylogenetic trees were estimated using the neighbour-joining method under the GTR+gamma model of nucleotide substitution, as implemented in PAUP* [3]. Reliability of clustering was assessed by bootstrap analysis (100 replicates). Further phylogenetic analysis within HIV-1 subtypes, included a large set of HIV-1 sequences from Greece sampled between 1998 and 2009 (more than 2,000 cases) and reference sequences sampled globally [4]. Only grouped sequences from IDUs that received bootstrap support higher than $75 \%$ were considered as 'clustered' [5].
According to the subtyping analysis, the prevalence of HIV-1 clades in the newly identified samples from IDUs in 2010 and 2011 was as follows: subtype $A:$ 20/34, subtype B: 9/34, subtype G: 4/34 and CRFo2_AG: 1/34 [3] (Table).

These figures were substantially different from the prevalence of HIV-1 subtypes estimated from 2,327 HIV-1 infected individuals sampled during the period from 1998 to 2009 , which comprise $24 \%$ of the total cases of HIV-1 infection reported in Greece since the beginning of the HIV epidemic (Table) $[4,6]$.

Further analysis of the subtype $\mathrm{G}$ sequences classified them as CRF14_BG, which belongs to subtype $G$ in the partial PR and RT region.

Detailed phylogenetic analyses, including a large set of Greek isolates sampled between 1998 and 2011 as well as reference isolates from other countries, revealed that 28 of the 34 sequences from the newly identified cases of HIV-1 infection among IDUs in 2010 and 2011 fell within seven separate phylogenetic clusters. More specifically, six of the 11 of the sequences from 2010 were found in three clusters, and 22 of the 23 the sequences from 2011 were found in four clusters.

Seventeen of the 20 individuals infected with subtype A fell in clusters of IDU local transmission networks. Among those, we identified three phylogenetic clusters consisting of 12 , three and two sequences. The cluster of 12 , shown as an example in Figure $2 \mathrm{~A}$, formed part of a larger cluster of sequences obtained from infected IDUs in Asia. Based on previous analyses of a large population of local viral isolates $(n=2,327)[6]$, this is the first identification of HIV-1 subtype A Asian strains in Greece. This finding supports a recent introduction from migrating population although alternative hypotheses cannot be entirely excluded. The two smaller clusters of subtype A were nested within a large population of IDUs from the Former Soviet Union countries.

For subtype $B$, six of nine sequences formed two phylogenetic clusters of, both originating from Greece (Figure 2B). One of the clusters contained five isolates, the sixth isolate was part of a cluster of two, one of which was sampled before 2010. For subtypes $G$ and CRFo2_AG, all cases were grouped in phylogenetic clusters of four and one sequence, respectively originating from southwest Europe.

\section{TABLE}

Prevalence of HIV-1 subtypes in the general population and in injecting drug users, Greece, 1998-2009 and 2010-2011

\begin{tabular}{|l|c|c|c|c|c|c|}
\hline \multirow{2}{*}{ Population } & \multicolumn{5}{|c|}{ Subtypes (n, \%) } \\
\cline { 2 - 7 } & $\mathrm{A}$ & $\mathrm{B}$ & $\mathrm{G}$ & CRFo2_AG & Others & \multicolumn{1}{|c|}{ Total } \\
\hline Total HIV-1 infected population sampled during 1998-2009 & $572(24.6 \%)$ & $\mathbf{1 , 3 9 6}(60.0 \%)$ & $\mathbf{2 0}(0.9 \%)$ & $44(1.9 \%)$ & $296(12.7 \%)$ & $\mathbf{2 , 3 2 7}$ \\
\hline Injecting drug users sampled during 2010-2011 & $20(58.8 \%)$ & $9(26.5 \%)$ & $4(11.8 \%)$ & $1(2.9 \%)$ & $0(0 \%)$ & $\mathbf{3 4}$ \\
\hline
\end{tabular}




\section{FIGURE 2}

Phylogenetic trees of HIV-1 isolates sampled in Greece and other countries between 1998 and 2011

A

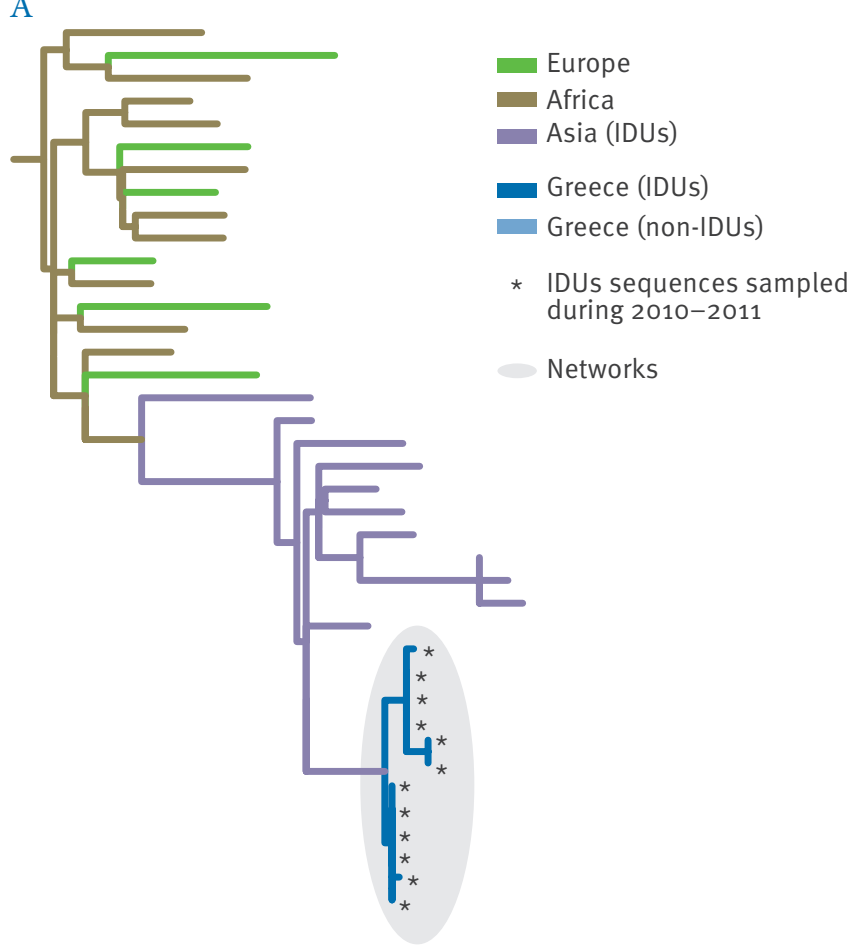

B

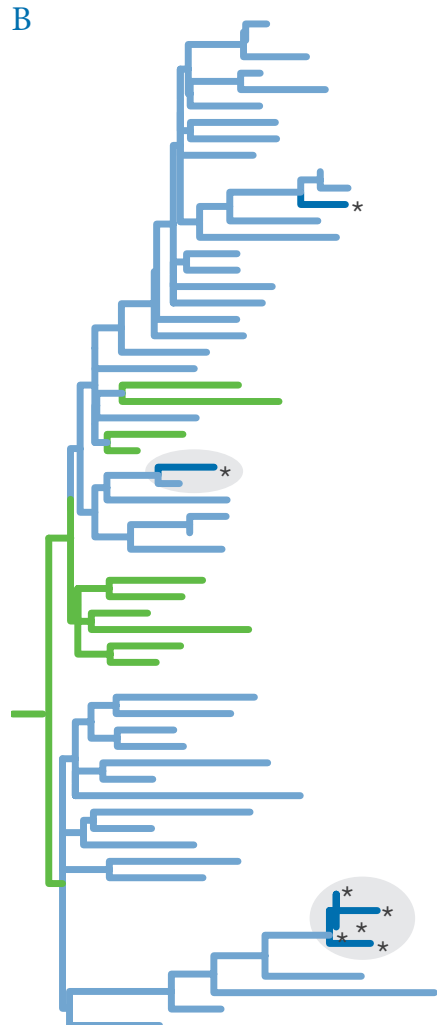

A: HIV-1 sequences from IDUs in Greece originating from Asia (subtype A).

B: HIV-1 sequences from IDUs in Greece originating from a local transmission network (subtype B).
Moreover, the branch lengths for all phylogenetic clusters identified in 2011 were very short, suggesting a very recent infection among the study population (Figure 2). Only in one case an additional sequence from 2010 (subtype G) was found within the shortbranched phylogenetic clusters from 2011.

\section{Discussion}

Until the beginning of 2011, the HIV-1 epidemic in Greece had been concentrated on MSM. Nevertheless, since 2010, the pattern of viral transmission in Greece seems to be changing now affecting also substantially the subgroup of IDUs. Data from the national HIV-1/AIDS registry showed an increase higher than 10 -fold in the rate of notified cases of HIV-1 in IDUs, which amounted to approximately one fifth of the total recorded cases for the first seven months of 2011.

Firstly, in accordance with surveillance data, our preliminary molecular epidemiology results indicated short-branched clusters in 2011, which were highly suggestive of a recent epidemic among the IDUs. Secondly, the prevalence of HIV-1 subtypes was different from previous estimates derived from a large population of HIV-1 infected individuals in Greece $[4,6]$. Thirdly, the new epidemic seems to be spreading through transmission networks of different sizes, suggesting a limited number of sources, or high levels of transmission networking among the IDUs. The largest transmission network consisted of 12 sequences including half of the analysed IDUs samples in 2011. According to the Greek HIV/AIDS molecular surveillance programme, these sequences derived from newly identified cases of HIV-1 infection. Fourthly, viral sources for the different networks were mainly originated from globally circulating viruses (CRF14_BG, subtype A) suggesting a potential role of migrant IDUs for the initiation of the recent outbreak $[7,8]$.

A potential limitation was the small number of HIV-1 sequences from IDUs included in the analysis. Final conclusions about the levels of networking will be made based on additional data as the outbreak evolves.

HIV infection is a serious consequence of drug use and remains an important public health challenge. The high prevalence of HIV-1 infection among the IDUs in the eastern part of Europe is still worrying [9]. Interestingly, a neighbouring country, Bulgaria, experienced a steady increase in HIV reporting rates, from none per million population in 2003 to almost seven per million population in 2008 [10]. Given the estimated large number of IDUs $(20,000-27,000)$ (unpublished data) who inject illicit drugs in Greece and the limited resources in the public sector because of the current financial situation in Greece, public health authorities face the potential of a rapidly growing HIV-1 epidemic in this vulnerable subset of population and, possibly to the wider community, with dramatic medical, social and economical consequences [11-13]. Preventive interventions and epidemiological monitoring along with an appropriate 
allocation of available resources are the key components of an effective and sustained response. An integrated and combined prevention initiative including awareness campaigns targeting IDUs and healthcare and social personnel working with IDUs, large-scale distribution of sterile injecting equipment, increased access to opioid substitution therapy, higher uptake of HIV-1 testing and expanded coverage of antiretroviral treatment among IDUs, have shown to be effective in decreasing transmission rates in IDUs. These should be taken into consideration as measures to stop the outbreak [14-19]. Otherwise, the authors believe that the HIV epidemic in Greece is potentially unstoppable.
16. Kerr T, Small W, Buchner C, Zhang R, Li K, Montaner J, et al. Syringe sharing and HIV incidence among injection drug users and increased access to sterile syringes. Am J Public Health. 2010;100(8):1449-53.

17. Strathdee SA, Hallett TB, Bobrova N, Rhodes T, Booth R, Abdool R, et al. HIV and risk environment for injecting drug users: the past, present, and future. Lancet. 2010;376(9737):268-84.

18. Wood E, Kerr T, Marshall BD, Li K, Zhang R, Hogg RS, et al. Longitudinal community plasma HIV-1 RNA concentrations and incidence of HIV-1 among injecting drug users: prospective cohort study. BMJ. 2009;338:b1649.

19. Uhlmann S, Milloy MJ, Kerr T, Zhang R, Guillemi S, Marsh D, et al. Methadone maintenance therapy promotes initiation of antiretroviral therapy among injection drug users. Addiction. 2010;105(5):907-13.

\section{References}

1. Nikolopoulos G, Paraskevis D, Hatzakis A. HIV epidemiology in Greece. Future Microbiol. 2008; 3(5):507-16.

2. Hellenic Center for Disease Control and Prevention (HCDCP). HIV/AIDS Surveillance Report in Greece, 31-10-2010. (Issue 25). HCDCP: Athens. 2010. Available from: http://www.hivaids.gr/ pliroforisi/epidiomiologik02010.pdf

3. Swofford DL. PAUP*. Phylogenetic Analysis Using Parsimony (*and Other Methods). Version 4. Sinauer Associates. Sunderland, Massachusetts. 1998.

4. Paraskevis D, Magiorkinis E, Magiorkinis G, Sypsa V, Paparizos V, Lazanas M, et al. Increasing prevalence of HIV-1 subtype A in Greece: estimating epidemic history and origin. J Infect Dis. 2007;196(8):1167-76.

5. Paraskevis D, Pybus O, Magiorkinis G, Hatzakis A, Wensing AM, van de Vijver DA, et al. Tracing the HIV-1 subtype B mobility in Europe: a phylogeographic approach. Retrovirology. 2009;6:49.

6. Paraskevis D M, Zavitsanou E, Detsika A, Magiorkinis M, Papa G, Beloukas A, et al for the multicenter study of HIV heterogeneity (2010). Molecular typing of the HIV-1 networks through a nationwide study in Greece: predominance of subtype A over B spreading among the natives. 10th International Conference on Molecular Epidemiology and Evolutionary Genetics of Infectious Diseases Amsterdam. 3-5 Nov 2010.

7. Delgado E, Thomson MM, Villahermosa ML, Sierra M, Ocampo A, Miralles C, et al. Identification of a newly characterized HIV-1 BG intersubtype circulating recombinant form in Galicia, Spain, which exhibits a pseudotype-like virion structure. J Acquir Immune Defic Syndr. 2002;29(5):536-43.

8. Bobkov A, Cheingsong-Popov R, Selimova L, Ladnaya N, Kazennova E, Kravchenko A, et al. An HIV type 1 epidemic among injecting drug users in the former Soviet Union caused by a homogeneous subtype A strain. AIDS Res Hum Retroviruses. 1997;13(14):1195-201.

9. Wiessing L, van de Laar MJ, Donoghoe MC, Guarita B, Klempová D, Griffiths P. HIV among injecting drug users in Europe: increasing trends in the East. Euro Surveill. 2008;13(50): pii=19067. Available from: http://www. eurosurveillance.org/ViewArticle.aspx?Articleld=19067

10. European Monitoring Centre for Drugs and Drug Addiction (EMCDDA). 2010 Annual report on the state of the drugs problem in Europe. Lisbon: EMCDDA. Nov 2010. Available from: http://www.emcdda.europa.eu/attachements.cfm/ att_120104_EN_EMCDDA_AR2010_EN.pdf

11. Des Jarlais DC, Semaan S. HIV prevention for injecting drug users: the first 25 years and counting. Psychosom Med. 2008;70(5):606-11.

12. Friedman SR, Rossi D, Braine N. Theorizing “Big Events" as a potential risk environment for drug use, drug-related harm and HIV epidemic outbreaks. Int J Drug Policy. 2009;20(3):283-91.

13. Mathers BM, Degenhardt L, Phillips B, Wiessing L, Hickman M, Strathdee SA, et al. Global epidemiology of injecting drug use and HIV among people who inject drugs: a systematic review. Lancet. 2008;372(9651):1733-45.

14. Bruce RD. Methadone as HIV prevention: high volume methadone sites to decrease HIV incidence rates in resource limited settings. Int J Drug Policy. 2010;21(2):122-4.

15. Degenhardt L, Mathers B, Vickerman P, Rhodes T, Latkin C, Hickman M. Prevention of HIV infection for people who inject drugs: why individual, structural, and combination approaches are needed. Lancet. 2010;376(9737):285-301. 